\title{
Sclerosing Epithelioid Fibrosarcoma
}

\section{Case Presentation and a Systematic Review}

\author{
Christian Ossendorf MD, Gabriela M. Studer MD, \\ Beata Bode MD, Bruno Fuchs MD, PhD
}

Received: 11 August 2007/Accepted: 20 February 2008/Published online: 14 March 2008

(C) The Association of Bone and Joint Surgeons 2008

\begin{abstract}
In sclerosing epithelioid fibrosarcoma (SEF), a rare variant of low-grade fibrosarcoma, treatment results and therapeutic options are poorly characterized. We systematically analyzed the data of all 89 patients ( 43 female, 46 male; mean age, 47 years [range, 14-87 years]) reported in the literature concerning clinical presentation, histopathology, differential diagnosis, treatment, survival rates, and prognosis, and we present an additional case. Information detailing treatment, disease control, and followup was available in 60 (67\%), 75 (84\%), and 68 patients (76\%), respectively. Case history was variable with one-third of patients reporting a painful, enlarging mass. Ten patients $(13 \%)$ presented with metastases, $23(31 \%)$ had metastases develop after diagnosis, and 28 (37\%) had local recurrence.
\end{abstract}

Each author certifies that he or she has no commercial associations (eg, consultancies, stock ownership, equity interest, patent/licensing arrangements, etc) that might pose a conflict of interest in connection with the submitted article.

Each author certifies that his or her institution has approved or waived approval for the reporting of this case and that all investigations were conducted in conformity with ethical principles of research.

Electronic supplementary material The online version of this article (doi:10.1007/s11999-008-0205-8) contains supplementary material, which is available to authorized users.

C. Ossendorf, B. Fuchs $(\square)$

Department of Orthopedics, Balgrist University Hospital, University of Zurich, Forchstr 340, 8008 Zurich, Switzerland e-mail: bruno.fuchs@balgrist.ch

\section{G. M. Studer}

Department of Radiation Oncology, University of Zurich, Zurich, Switzerland

B. Bode

Institute of Surgical Pathology, University of Zurich, Zurich, Switzerland
Low cellularity, mild pleomorphy, and sclerotic hyaline matrix of SEF suggest a benign clinical behavior, and cell morphology allows for the wide differential diagnosis of benign, pseudosarcomatous, and malignant proliferations. In addition to surgery, 11 patients (15\%) had chemotherapy, $22(29 \%)$ had postoperative radiation therapy, and three (4\%) had a combination of both. Twenty-three patients (34\%) died from their disease after a mean of 46 months, 24 $(35 \%)$ were alive with disease, and $20(31 \%)$ were alive without evidence of disease. Patients with SEF of the head and neck had the worst prognosis.

Level of Evidence: Level III, prognostic study. See the Guidelines for Authors for a complete description of levels of evidence.

\section{Introduction}

Sclerosing epithelioid fibrosarcoma is a rare and poorly recognized but distinct variant of fibrosarcoma (ICD-O code 88 10/3). Since it was originally published in 1995 by Meis-Kindblom et al. in a series of 25 patients [36], 25 reports on 89 patients with a main focus on histopathologic and immunohistochemical features have been reported $[1,2,4-7,9,10,12-16,18,22,23,25,29-32,36,40,42$, 43]. These reports suggest SEF is characterized histopathologically by low-grade tumor features: round or oval cells typically arranged in a cord- or nest-like distribution with a collagen background almost effacing the neoplastic cells. Mitotic figures are either scant or absent, and necrosis is uncommon. Sclerosing epithelioid fibrosarcoma is characterized by rearrangement of 10p11 [25, 40] and amplification of 12q13 and 12q15, including the HMGIC gene, a transcriptional activator [16]. Together with lowgrade fibromyxoid sarcoma (FMS) and hyalinizing spindle 
cell tumor with giant rosettes (HSCTGR) [3], SEF belongs to the family of fibrosing fibrosarcomas [44, 45]. Each of these tumors has distinctive clinical features; they share in common histologic components suggesting a close relationship [3, 20, 21, 42, 43].

The response of SEF to treatment has not yet been fully characterized. Furthermore, the current low-grade classification might be misleading because it disregards the full malignant potential of SEF. The resulting lack of familiarity of treating physicians with SEF might lead to misinterpretation of this rare entity. Thus, the malignant potential of SEF might be underestimated. The potential consequence of this is inadequate therapy leading to an unfavorable treatment result.

Consequently, we systematically reviewed the literature to highlight the following questions: Is there a typical clinical presentation of SEF (patient history, tumor size and location, distant tumor manifestation, and rate of metastasis)? What are the histopathologic features of SEF and what differential diagnoses arise? How were patients with SEF treated and how does this therapy influence overall, disease-free survival and prognosis? Does distant disease depend on tumor size? How do location of the primary tumor, local treatment, and gender influence the prognosis?

\section{Materials and Methods}

We performed a systematic review of all SEF studies and case presentations published until December 22, 2007. All publications were derived from NCBI-PubMed (www. pubmed.gov) and Embase (www.embase.com) using the search term sclerosing epithelioid fibrosarcoma. Together, this yielded 34 articles, of which 25 were primarily about SEF. We included all these 25 studies in the current review and analyzed them in detail. The remaining nine studies concern articles about fibromyxoid sarcoma [41] or soft tissue tumor pathology [24, 37], low-grade fibrosarcoma not otherwise specified [28], rhabdomyosarcoma [20], deep fibromatosis [8], or mention SEF only marginally [21, 27, 33]. Thus, we did not include these studies in our review.

Since 1995, 89 patients (not including our patient) were evaluated in these 25 studies regarding tumor characteristics, treatment, and clinical outcome $[1,2,4-7,9,10,12$ $16,18,22,23,25,29-32,36,40,42,43]$. Information detailing treatment, disease control, and followup was available in 60 (67\%), 75 (84\%), and 68 patients (76\%), respectively (Supplemental Website Material; a supplemental table is available with the online version of CORR). Including our case, tumors were $8 \mathrm{~cm}$ on average and the mean patient age was 47 years (range, 14-87 years) (Table 1). The mean duration between first signs or symptoms and diagnosis was 33 months on average (range, 1 month-13 years). Sixty patients underwent surgery, which included biopsy only $(\mathrm{n}=2[3 \%])$; not further specified resection/unspecified surgery $(\mathrm{n}=25[42 \%])$; amputation $(\mathrm{n}=5$ [8\%]); and wide $(\mathrm{n}=21 \quad[35 \%])$, marginal, and intralesional excisions $(n=7$ [12\%]). In eight patients, information regarding age was not provided. Sixteen of 82 patients (19\%) were younger than 30 years at diagnosis, 30 patients (37\%) were 30 to 50 years old, and 36 patients $(44 \%)$ were older than 50 years. Study quality was judged according to the levels of evidence based on the US Preventive Services Task Force for ranking evidence regarding the effectiveness of treatments or screening as presented by Clinical Orthopaedics and Related Research (http://www.editorialmanager.com/corr/).

We tested the influence of gender on prognosis of SEF using the Wilcoxon rank sum test. We used SPSS for Windows (SPSS, Chicago, IL). A value of $p<0.05$ was considered statistically significant.

\section{Case Report}

A 32-year-old woman presented with an 18-month history of increasing swelling at her right distal lateral forearm, which initially was painless. Conventional radiographs

Table 1. Patients' characteristics

\begin{tabular}{llll}
\hline Characteristic & $\begin{array}{l}\text { Number of patients } \\
\text { in whom information } \\
\text { was provided per } \\
\text { study population }\end{array}$ & Mean & Quality \\
\hline Gender & $90 / 90$ & & 44 females, 46 males \\
Age & $82 / 90$ & 47 years (range, 14-87 years) & \\
History (months) & $23 / 90$ & 33 years (range, 1 month-13 years) & \\
$\begin{array}{l}\text { Size (cm) } \\
\text { Surgery }\end{array}$ & $78 / 90$ & 8.3 years (range, 1-25 years) & $\begin{array}{l}\text { Biopsy only (n }=2) ; \text { not further specified } \\
\text { resection/unspecified surgery }(\mathrm{n}=25) ; \\
\text { amputation }(\mathrm{n}=5) ; \text { wide excision }(\mathrm{n}=21), \\
\text { marginal and intralesional excision }(\mathrm{n}=7)\end{array}$ \\
\end{tabular}




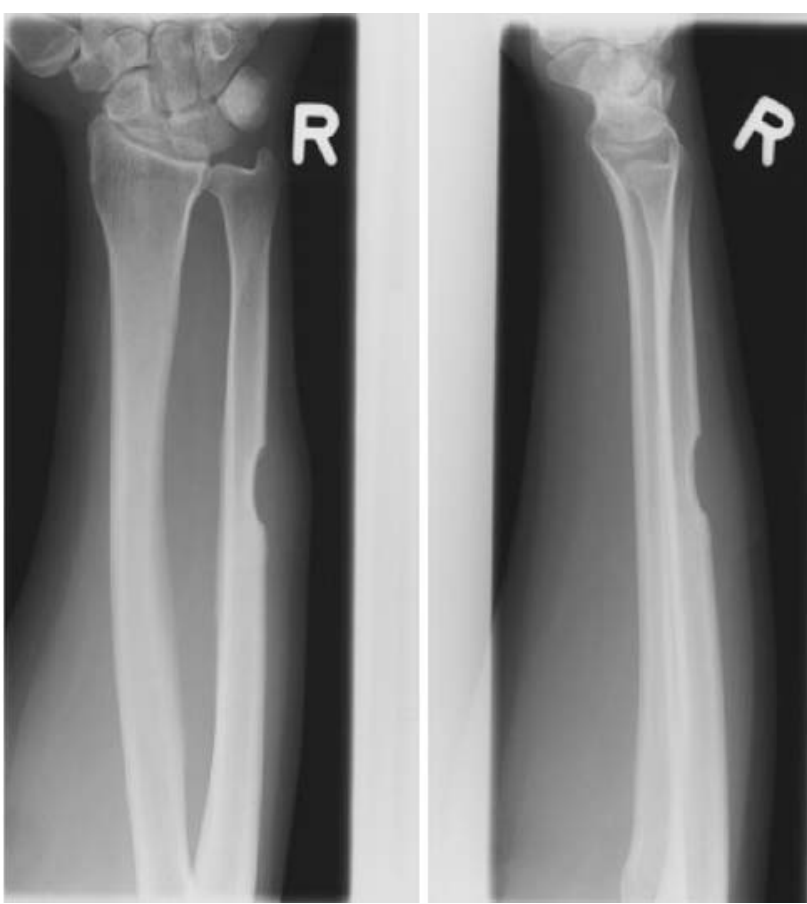

Fig. 1 (A) Anteroposterior and (B) lateral radiographs show a punched-out osteolytic lesion surrounded by a sclerotic rim at the middiaphysis of the right ulna.

showed a punched-out osteolytic lesion surrounded by a sclerotic rim at the ulna (Fig. 1). Magnetic resonance imaging revealed a $5 \times 1.5-\mathrm{cm}$ tumor and some edema of the bone marrow (Fig. 2).

We performed a core needle biopsy and after establishing the diagnosis, performed complete gross resection with positive microscopic resection margins. Because of tumor infiltration, the extensor carpi ulnaris muscle and parts of the adjacent muscles were removed. Subsequently, we used a tricortical autologous bone graft from the iliac crest for reconstruction. A 3.5-mm low-contact dynamic compression plate was used to fix the bone graft and to provide stability to the ulna (Fig. 3).

Histologically, there was a sharply demarcated tumor mass in the direct vicinity of the periosteum with infiltration of the cortical bone. The tumor tissue consisted of highly collagenized extracellular matrix with a low density of cells arranged in loose bundles. Most of the cells were elongated with a spindle-shaped nucleus and abundant eosinophilic cytoplasm (Fig. 3A). Focally, particularly at the periphery of the tumor, there were somewhat more cellular areas with plump, epithelioid cells of variable size and individual large, pleomorphic nuclei with prominent nucleoli (Fig. 3B). We detected no mitotic figures or areas of necrosis. Immunohistochemically, no reactivity was detected to any of the tested epithelial, myogenic, or neural markers (actin, desmin, myogenin, S-100, cytokeratin, beta-catenin, CD 31, CD 34, CD 99, CD 117, EMA, bcl-2,

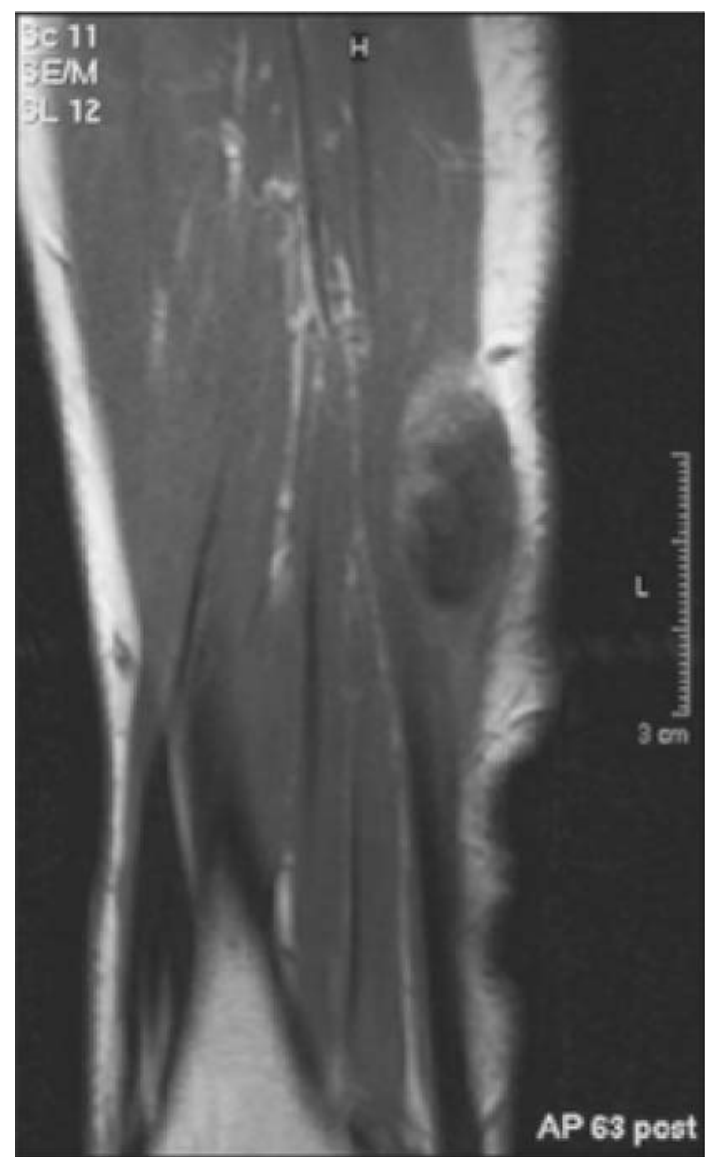

Fig. 2 An MRI scan of the right forearm shows a $5 \times 1.5-\mathrm{cm}$ tumor extending into the soft tissues and slight bone marrow edema.

MDM-2, MIB-1) with sole positivity for the relatively unspecific, mesenchymal intermediate filament vimentin (Fig. 3C). Thus, we established the diagnosis of a SEF on morphologic criteria.

Subsequently, we administered adjuvant radiation therapy (66 Gy, 1.8 Gy per session). No systemic treatment was given.

Two years postoperatively, imaging studies, including computed tomography of the chest and MRI of the right forearm, confirmed no local tumor and no evidence of systemic disease.

\section{Results}

Based on our literature review, the clinical behavior of SEF was generally aggressive with recurrence after resection in 27 of the 90 patients (30\%) at the various times of reported followups. One patient had persistent disease and in 15 patients $(17 \%)$, information regarding recurrence was not provided by the respective authors. Tumor size at diagnosis averaged $8.3 \mathrm{~cm}$ (range, $1-25 \mathrm{~cm}$ ). Most patients had a 


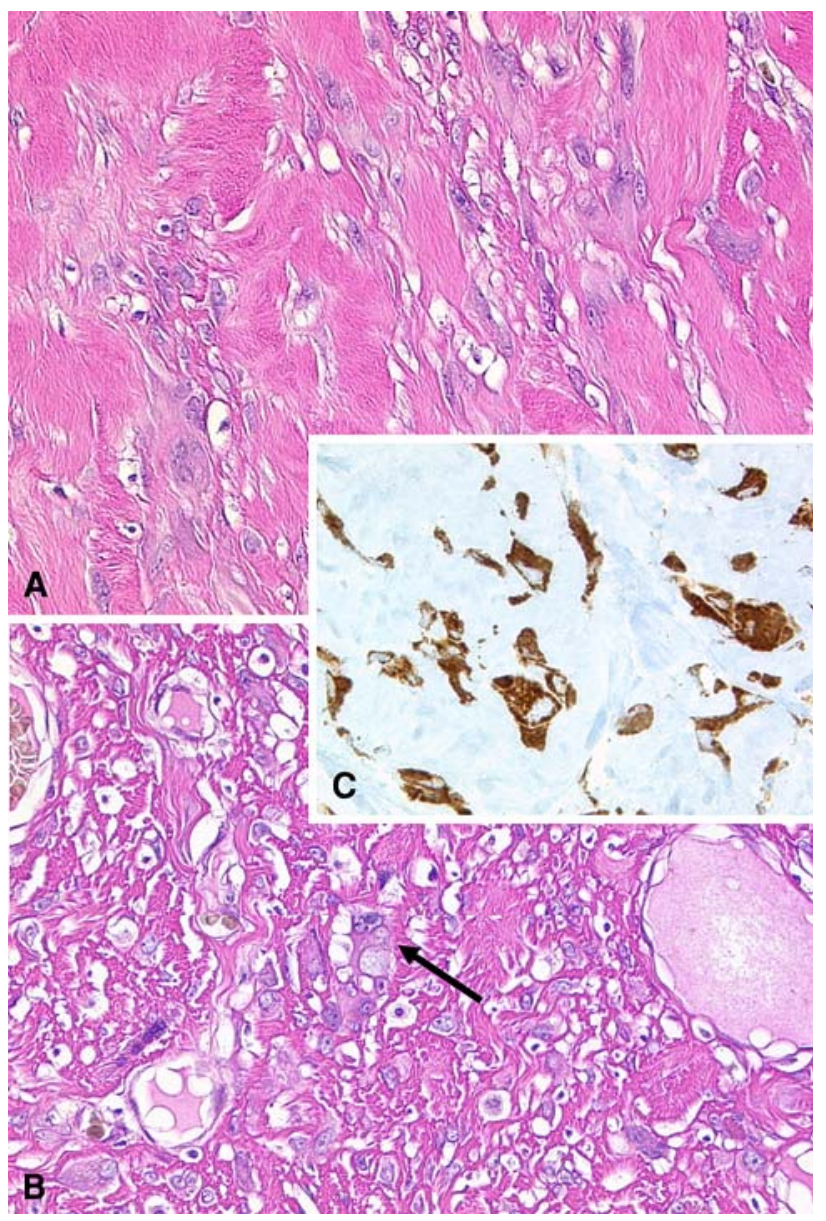

Fig. 3A-C (A) Histologically, the tumor consists of extensive areas with high collagen content and scarce mesenchymal cells (Stain, hematoxylin \& eosin; original magnification, $\times 400$ ). (B) At the tumor periphery, there are areas with higher cellularity and more pronounced cell atypia (arrow) (Stain, hematoxylin \& eosin; original magnification, $\times 200$ ). (C) The tumor cells are immunohistochemically positive for the mesenchymal marker vimentin (Dako, Baar, Switzerland; antibody dilution 1:250) (original magnification, $\times 400$ ).

variable case history with one-third reporting a painful and enlarging mass, mostly a deep muscular lesion associated with the adjacent fascia or periosteum. The most frequent tumor sites were the lower extremities/limb girdle $(\mathrm{n}=35$ [39\%]; average tumor size, $9.37 \mathrm{~cm})$ and trunk $(\mathrm{n}=19$ [21\%]; average tumor size, $7.60 \mathrm{~cm}$ ) followed by the upper extremities $(\mathrm{n}=13 \quad[14.5 \%]$; average tumor size, $7.65 \mathrm{~cm})$, the head and neck $(\mathrm{n}=13[14.5 \%]$; average tumor size, $6.69 \mathrm{~cm})$, and abdominal/inguinal $(\mathrm{n}=10$ [11\%]; average tumor size, $9.39 \mathrm{~cm}$ ). Overall, 75 of 90 patients $(83 \%)$ were reported to have distant disease at a mean of 36 months after diagnosis. Ten of 37 patients (27\%) presented with metastases at the time of the primary diagnosis. The most frequent site of distant tumor manifestation was the lung $(\mathrm{n}=26$ [70\%]) followed by osseous lesions to multiple bones $(\mathrm{n}=15[41 \%])$ and to the pleura/ chest wall $(\mathrm{n}=4[11 \%])$. Two patients had metastases to the scalp (5\%). Lymphatic spread, metastases to the breast and to the liver were reported in one patient (3\%). Fourteen patients (38\%) had metastases to multiple sites. None of the patients with bone involvement had a bone tumor elsewhere.

In SEF, the deceptively low cellularity, mild pleomorphy, and the densely sclerotic hyaline matrix may suggest a benign clinical behavior. Cell morphology allows for the wide differential diagnosis of benign, pseudosarcomatous, and malignant proliferations. The typical morphologic features of sarcomas such as pronounced pleomorphy, infiltrative growth pattern, necrosis, and mitotic activity may be inconspicuous or even absent. These include nodular fasciitis, myositis ossificans, fibromatosis, hyalinizing leiomyoma, fibrous histiocytoma, lobular or signet ring cell carcinoma, sclerosing lymphoma, granulocytic sarcoma, ossifying fibromyxoid tumor, synovial sarcoma, or small cell osteosarcoma.

One patient received preoperative radiation therapy (50 Gy). Twenty-one patients (37\%) received postoperative radiation therapy (50.4-70.2 Gy) and 10 of 22 patients $(45 \%)$ received indicated radiation therapy doses. Ten of 56 patients (18\%) received chemotherapy, and three patients underwent combined radiation therapy and chemotherapy. Twenty-seven patients $(36 \%)$ experienced local recurrence after a mean of 36 months (range, 2132 months) after diagnosis and treatment. In each age subgroup, there were $50 \%$ to $60 \%$ of patients who were alive with disease or who had died of disease (DOD).

Distant disease seemed independent of tumor size (nine of 23 tumors [39\%] with $5 \mathrm{~cm}$ or less, 15 of 33 tumors [45\%] with $5-10 \mathrm{~cm}$, and 10 of 22 with $10 \mathrm{~cm}$ [45\%] or greater metastasized). However, no patient with a primary tumor of $5 \mathrm{~cm}$ or less initially presented with metastases. Tumors of patients with metastasis at diagnosis $(\mathrm{n}=10)$ were larger $(11.3 \mathrm{~cm}$; range, $7.5-17 \mathrm{~cm})$ than tumors that subsequently metastasized $(\mathrm{n}=23 ; 7.7 \mathrm{~cm}$; range, $2.5-19.5 \mathrm{~cm})$ and tumors of patients without metastasis $(\mathrm{n}=43 ; 8 \mathrm{~cm}$; range, $1-25 \mathrm{~cm})$. Forty-six percent (17 of 37 patients) of patients with metastases experienced local recurrence, and $63 \%$ of patients with local recurrence (17 of 27 patients) had distant metastases develop. Seven of 11 patients who underwent systemic chemotherapy had systemic disease develop.

With respect to location, patients with SEF of the head and neck had the worst prognosis with six of 13 patients (46\%) DOD followed by SEF of the upper extremities (five of 13 [38\%]) and the trunk (five of 19 [26\%]). The influence of local treatment could not be assessed adequately because of incompletely reported data regarding surgical procedures, resection status, followup, and missing criteria for radiotherapy. 
Sclerosing epithelioid fibrosarcoma of the upper extremities had worse distant control compared with SEF located in the lower extremities with metastasis in six of seven patients. Gender did not seem to influence $(p=0.069)$ local recurrence (13 male and 15 female patients). Followup was available in 16 of 22 patients who received radiation. However, in four and seven of 16 cases, resection status and radiotherapeutic doses were unknown. Twenty-three patients $(34 \%)$ died from their disease after a mean of 46 months. Twenty-four (35\%) were alive with disease and $20(31 \%)$ were alive without evidence of disease.

\section{Discussion}

We systematically reviewed the literature about SEF to determine its clinical presentation, histopathology, differential diagnosis, treatment, survival rates, and prognosis. Although histopathologic appearance of SEF suggests low grade, it is a high-grade tumor clinically.

The lack of control groups and prospective design, small sample sizes, and missing treatment details in the reviewed studies limit our interpretations. Because of incomplete data, we could not thoroughly assess the influence of surgery evaluated. The small number of patients in distinct subgroups did not afford adequate power for statistical analysis.

Sclerosing epithelioid fibrosarcoma, together with FMS and HSCTGR, belongs to the family of fibrosing fibrosarcomas. Fibromyxoid sarcoma initially was described by Evans [17] in 1987 and characterized as an indolent, lowgrade fibromyxoid neoplasm arising in deep soft tissue with paradoxical aggressive features. Hyalinizing spindle cell tumor with giant rosettes is a similarly rare low-grade fibrosarcoma variant with a very low metastatic potential. In FMS, the local failure rate was $33 \%$, metastases occurred in $42 \%$, and $18 \%$ of patients were DOD [18]. Local recurrence rates between $30 \%$ and $75 \%$ have been observed in several small series with followups ranging from $74 \%$ [38] to $100 \%$ [7]. In contrast, recurrence of metastasis ranged from 0\% [39] to 58\% [26]. Died of disease rates ranged from $14 \%$ and $33 \%$ [16]. Hyalinizing spindle cell tumor with giant rosettes was described for the first time by Lane et al. in 1997 [34], who reported one local recurrence and no metastases in 12 of 19 patients with available followup. So far, six patients with pulmonary metastases have been described $[11,19,20,35$, $38,45]$.

Hyalinizing spindle cell tumors with giant rosettes and FMS have histologic low-grade tumor features and HSCTGR often manifests by an indolent clinical behavior. Based on a series of 56 patients with a mean followup of 3 years, local failure occurred in five (9\%) and metastatic disease in three patients (6\%; one HSCTGR, two FMS, but no metastases at diagnosis). One patient was DOD (2\%). These data support the hypothesis that FMS and HSCTGR may represent the same entity and should be regarded as lowgrade sarcomas [20]. In contrast to HSCTGR and FMS, local recurrence in patients with SEF was seen in $40 \%$ and distant disease in $80 \%$, and $67 \%$ of patients with local recurrence had distant metastasis develop. Thus, SEF bears malignant potential and therefore appears as the most malignant variant in this family of low-grade fibrosarcomas [20, 21, 34, 42, 43] followed by FMS. This should be taken into consideration when the decision is made on a treatment plan.

Diagnostically, the most challenging problem is to distinguish SEF from undifferentiated carcinoma, which might be impossible based purely on histology. Therefore, immunohistochemical analysis appears mandatory. Specific protein expression or genetic changes have not been described in SEF. The only immunostaining marker consistently reported positive (as in our patient) was vimentin, which is a general marker for soft tissue sarcomas and therefore not specific for SEF $[1,3,4,6,7,9,10,13,18$, 29-32, 36, 40, 43]. Occasional positivity of rare tumor cells for other general markers of soft tissue sarcomas like EMA, S100, or cytokeratin are not useful for diagnosis of a patient with SEF, because these also may be positive in other tumors.

Sclerosing epithelioid fibrosarcoma is a clinically aggressive but histologically low-grade sarcoma with unsatisfactory treatment results. It typically takes 33 months from the first onset of symptoms to diagnosis. Sclerosing epithelioid fibrosarcoma can occur from adolescence to the older years and is localized mainly in soft tissues, but it also can arise as a primary tumor of bone, in the neural system, or in the ovary [40]. Sclerosing epithelioid fibrosarcoma has a predisposition for local recurrence with metastasis primarily to the lung. The role of systemic treatment remains unclear. Consequently, SEF may be treated preferably by resection, including reexcision after intralesional excision. Moreover, preoperative or postoperative radiation as used in other soft tissue sarcomas also should be considered.

Acknowledgments We thank Felix Regenfelder, MD, for advice and assistance in performing statistics.

\section{References}

1. Abdulkader I, Cameselle-Teijeiro J, Fraga M, Caparrini A, Forteza J. Sclerosing epithelioid fibrosarcoma primary of the bone. Int J Surg Pathol. 2002;10:227-230.

2. Antonescu CR, Baren A. Spectrum of low-grade fibrosarcomas: a comparative ultrastructural analysis of low-grade myxofibrosarcoma and fibromyxoid sarcoma. Ultrastruct Pathol. 2004;28: $321-332$. 
3. Antonescu CR, Erlandson RA. Fibrosarcoma mimicking plasmacytoma or carcinoma: an ultrastructural study of 4 cases. Ultrastruct Pathol. 2001;25:31-37.

4. Arnould L, Jouannelle C, Mege F, Maillefert F, Fargeot P, Devillebichot C, Collin F. [Sclerosing epithelioid fibrosarcoma: a fibrosarcoma with a very long course.]. Ann Pathol. 2000;20: 154-157.

5. Arya M, Garcia-Montes F, Patel HR, Emberton M, Mundy AR. A rare tumour in the pelvis presenting with lower urinary symptoms: 'sclerosing epithelioid fibrosarcoma'. Eur J Surg Oncol. 2001;27:121-122.

6. Battiata AP, Casler J. Sclerosing epithelioid fibrosarcoma: a case report. Ann Otol Rhinol Laryngol. 2005;114:87-89.

7. Bezic J, Tomic S, Glavina-Durdov M, Alfirevic D, Samija I, Krizanac S. Sclerosing epitheloid fibrosarcoma: a report of two cases. Pathologica. 2004;96:433-435.

8. Bhattacharya B, Dilworth HP, Iacobuzio-Donahue C, Ricci F, Weber K, Furlong MA, Fisher C, Montgomery E. Nuclear betacatenin expression distinguishes deep fibromatosis from other benign and malignant fibroblastic and myofibroblastic lesions. Am J Surg Pathol. 2005;29:653-659.

9. Bilsky MH, Schefler AC, Sandberg DI, Dunkel IJ, Rosenblum MK. Sclerosing epithelioid fibrosarcomas involving the neuraxis: report of three cases. Neurosurgery. 2000;47:956-959; discussion 959-960.

10. Boudova L, Michal M, Kinkor Z, Bencik V, Husek K, Mukensnabl P, Rousarova M. [Sclerosing epithelioid fibrosarcoma.] Cesk Patol. 2001;37:158-162.

11. Chang E, Lee A, Lee E, Shin O, Kang C, Kim JM, Chu YC. Hyalinizing spindle cell tumor with giant rosettes with pulmonary metastasis after a long hiatus: a case report. J Korean Med Sci. 2004;19:619-623.

12. Choi HY, Kwon NS, Lee SJ, Lee DK, Park K, Lee CW, Jo MK. Sclerosing epithelioid fibrosarcoma of the kidney. Korean $K$ Urol. 2007;48:986-989.

13. Chow LT, Lui YH, Kumta SM, Allen PW. Primary sclerosing epithelioid fibrosarcoma of the sacrum: a case report and review of the literature. J Clin Pathol. 2004;57:90-94.

14. Christensen DR, Ramsamooj R, Gilbert TJ. Sclerosing epithelioid fibrosarcoma: short T2 on MR imaging. Skeletal Radiol. 1997; 26:619-621.

15. Decroisette C, Melloni B, Moldovan D, Gazaille V, Fermeaux V, Bertin F, Bonnaud F. [Pleural metastases of sclerosing epithelioid fibrosarcoma] [in French]. Rev Pneumol Clin. 2006;62: 179-182.

16. Donner LR, Clawson K, Dobin SM. Sclerosing epithelioid fibrosarcoma: a cytogenetic, immunohistochemical, and ultrastructural study of an unusual histological variant. Cancer Genet Cytogenet. 2000;119:127-131.

17. Evans HL. Low-grade fibromyxoid sarcoma: a report of two metastasizing neoplasms having a deceptively benign appearance. Am J Clin Pathol. 1987;88:615-619.

18. Eyden BP, Manson C, Banerjee SS, Roberts IS, Harris M. Sclerosing epithelioid fibrosarcoma: a study of five cases emphasizing diagnostic criteria. Histopathology. 1998;33:354-360.

19. Farinha P, Oliveira P, Soares J. Metastasizing hyalinizing spindle cell tumour with giant rosettes: report of a case with long survival. Histopathology. 2000;36:92-93.

20. Folpe AL, Lane KL, Paull G, Weiss SW. Low-grade fibromyxoid sarcoma and hyalinizing spindle cell tumor with giant rosettes: a clinicopathologic study of 73 cases supporting their identity and assessing the impact of high-grade areas. Am J Surg Pathol. 2000;24:1353-1360.

21. Franchi A, Massi D, Santucci M. Hyalinizing spindle cell tumor with giant rosettes and low-grade fibromyxoid sarcoma: an immunohistochemical and ultrastructural comparative investigation. Ultrastruct Pathol. 2003;27:349-355.

22. Frattini JC, Sosa JA, Carmack S, Robert ME. Sclerosing epithelioid fibrosarcoma of the cecum: a radiation-associated tumor in a previously unreported site. Arch Pathol Lab Med. 2007;131: $1825-1828$.

23. Garcia de León MA, Torres Coscoyuela M, Hernández Sánchez L. Sclerosing epithelioid fibrosarcoma: a complicated diagnosis. Rev Orthop Traumatol. 2001;45:419-422.

24. Genevay M, Coindre JM, Guillou L. [Recent entities in soft tissue tumor pathology. Part 2][in French]. Ann Pathol. 2003;23: $135-148$.

25. Gisselsson D, Andreasson P, Meis-Kindblom JM, Kindblom LG, Mertens F, Mandahl N. Amplification of $12 \mathrm{q} 13$ and $12 \mathrm{q} 15$ sequences in a sclerosing epithelioid fibrosarcoma. Cancer Genet Cytogenet. 1998;107:102-106.

26. Goodlad JR, Mentzel T, Fletcher CD. Low grade fibromyxoid sarcoma: clinicopathological analysis of eleven new cases in support of a distinct entity. Histopathology. 1995;26:229-237.

27. Guillou L, Benhattar J, Gengler C, Gallagher G, Ranchere-Vince D, Collin F, Terrier P, Terrier-Lacombe MJ, Leroux A, Marques B, Aubain Somerhausen Nde S, Keslair F, Pedeutour F, Coindre JM. Translocation-positive low-grade fibromyxoid sarcoma: clinicopathologic and molecular analysis of a series expanding the morphologic spectrum and suggesting potential relationship to sclerosing epithelioid fibrosarcoma: a study from the French Sarcoma Group. Am J Surg Pathol. 2007;31:1387-1402.

28. Hansen T, Katenkamp K, Brodhun M, Katenkamp D. Low-grade fibrosarcoma: report on 39 not otherwise specified cases and comparison with defined low-grade fibrosarcoma types. Histopathology. 2006;49:152-160.

29. Hanson IM, Pearson JM, Eyden BP, Slawik S, Harris M. Evidence of nerve sheath differentiation and high grade morphology in sclerosing epithelioid fibrosarcoma. J Clin Pathol. 2001;54:721-723.

30. Hindermann W, Katenkamp D. [Sclerosing epithelioid fibrosarcoma][in German]. Pathologe. 2003;24:103-108.

31. Hu WW, Lai RQ, Wang J, Feng XD, Li GM. [Sclerosing epithelioid fibrosarcoma: a clinicopathologic study of eight cases][in Chinese]. Zhonghua Bing Li Xue Za Zhi. 2004;33:337-341.

32. Jiao J, Rhodes DG, Burgess DJ. Multiple emulsion stability: pressure balance and interfacial film strength. J Colloid Interface Sci. 2002;250:444-450.

33. Karaki S, Mochida J, Lee YH, Nishimura K, Tsutsumi Y. Lowgrade malignant perineurioma of the paravertebral column, transforming into a high-grade malignancy. Pathol Int. 1999; $49: 820-825$.

34. Lane KL, Shannon RJ, Weiss SW. Hyalinizing spindle cell tumor with giant rosettes: a distinctive tumor closely resembling lowgrade fibromyxoid sarcoma. Am J Surg Pathol. 1997;21:14811488 .

35. Magro G, Fraggetta F, Manusia M, Mingrino A. Hyalinizing spindle cell tumor with giant rosettes: a previously undescribed lesion of the lung. Am J Surg Pathol. 1998;22:1431-1433.

36. Meis-Kindblom JM, Kindblom LG, Enzinger FM. Sclerosing epithelioid fibrosarcoma: a variant of fibrosarcoma simulating carcinoma. Am J Surg Pathol. 1995;19:979-993.

37. Miettinen M. From morphological to molecular diagnosis of soft tissue tumors. Adv Exp Med Biol. 2006;587:99-113.

38. O'Sullivan MJ, Sirgi KE, Dehner LP. Low-grade fibrosarcoma (hyalinizing spindle cell tumor with giant rosettes) with pulmonary metastases at presentation: case report and review of the literature. Int J Surg Pathol. 2002;10:211-216.

39. Oda Y, Takahira T, Kawaguchi K, Yamamoto H, Tamiya S, Matsuda S, Tanaka K, Iwamoto Y, Tsuneyoshi M. Low-grade 
fibromyxoid sarcoma versus low-grade myxofibrosarcoma in the extremities and trunk: a comparison of clinicopathological and immunohistochemical features. Histopathology. 2004;45:29-38.

40. Ogose A, Kawashima H, Umezu H, Hotta T, Gu W, Yamagiwa $\mathrm{H}$, Ito T, Tohyama T, Nishijima H, Endo N. Sclerosing epithelioid fibrosarcoma with $\operatorname{der}(10) \mathrm{t}(10 ; 17)(\mathrm{p} 11 ; \mathrm{q} 11)$. Cancer Genet Cytogenet. 2004;152:136-140.

41. Perigny $M$, Dion $N$, Couture $C$, Lagace R. [Low grade fibromyxoid sarcoma: a clinico-pathologic analysis of 7 cases] [in French]. Ann Pathol. 2006;26:419-425.
42. Reid R, Barrett A, Hamblen DL. Sclerosing epithelioid fibrosarcoma. Histopathology. 1996;28:451-455.

43. Watanabe K, Suzuki T. Epithelioid fibrosarcoma of the ovary. Virchows Arch. 2004;445:410-413.

44. Weiss SW, Goldblum JR. Enzinger and Weiss' Soft Tissue Tumors. 4th ed. St Louis MO: Mosby; 2001.

45. Woodruff JM, Antonescu CR, Erlandson RA, Boland PJ. Lowgrade fibrosarcoma with palisaded granulomalike bodies (giant rosettes): report of a case that metastasized. Am J Surg Pathol. 1999;23:1423-1428. 